Europhysics Letters

PREPRINT

\title{
Shaking a box of sand
}

\author{
P.F. Stadler ${ }^{1}(*)$, J.M. LuCK $\left.{ }^{2}{ }^{* *}\right)$ and Anita Mehta $\left.{ }^{3}{ }^{* * *}\right)$ \\ 1 Institut für Theoretische Chemie und Molekulare Strukturbiologie, Universität Wien, \\ Währingerstraße 17, A-1090 Wien, Austria; and The Santa Fe Institute, 1399 Hyde \\ Park Rd., Santa Fe NM 87501, USA. \\ 2 Service de Physique Théorique( $\left.{ }^{* *}\right)$, CEA Saclay, 91191 Gif-sur-Yvette cedex, France. \\ 3 S N Bose National Centre for Basic Sciences, Block JD Sector 3, Salt Lake, Calcutta \\ 700098, India; and ICTP, Strada Costiera 11, 34100 Trieste, Italy.
}

PACS. 45.70.Cc - Static sandpiles; granular compaction.

PACS. 45.70.Mg - Granular flow: mixing, segregation and stratification.

\begin{abstract}
We present a simple model of a vibrated box of sand, and discuss its dynamics in terms of two parameters reflecting static and dynamic disorder respectively. The fluidised, intermediate and frozen ('glassy') dynamical regimes are extensively probed by analysing the response of the packing fraction to steady, as well as cyclic, shaking, and indicators of the onset of glassy behaviour are analysed. In the 'glassy' regime, our model is exactly solvable, and allows for the qualitative description of ageing phenomena in terms of two characteristic lengths; predictions are also made about the influence of grain shape anisotropy on ageing behaviour.
\end{abstract}

Vibrating sand results in very varied dynamics, ranging from glassy [1, 2] to fluidised [3, 4]. Recent experiments, e.g. [5], have gone some way in validating the notion [6] that its essential features are captured by models which incorporate the fast relaxation of individual particles together with the cooperative rearrangements of clusters. In this Letter we present a simple model of a vibrated sand-box, which interpolates between the glassy and fluidised regimes, and is based on the generalisation of an earlier cellular automaton (CA) model [7] of an avalanching sandpile. Our model shows both fast and slow dynamics in the appropriate regimes: in particular, it reduces to an exactly solvable model in the frozen ('glassy') regime, and provides one with a toy model for ageing in vibrated sand 8,9].

We consider a rectangular lattice of height $H$ and width $W$ with $N \leq H W$ grains located at its lattice points, shaken with vibration intensity $\Gamma$. Each 'grain' is a rectangle with sides 1 and $a \leq 1$, respectively. Consider a grain $(i, j)$ in row $i$, column $j$ whose height at any given time is given by $h_{i j}=n_{i j-}+a n_{i j+}$, with $n_{i j-}$ the number of vertical grains and $n_{i j+}$ the number of horizontal grains below $(i, j)$ :

(*) E-mail: studla@tbi.univie.ac.at

(**) E-mail: luck@spht.saclay.cea.fr

(***) E-mail: anita@boson.bose.res.in

$\left(*^{* *}\right)$ URA 2306 of CNRS

(C) EDP Sciences 


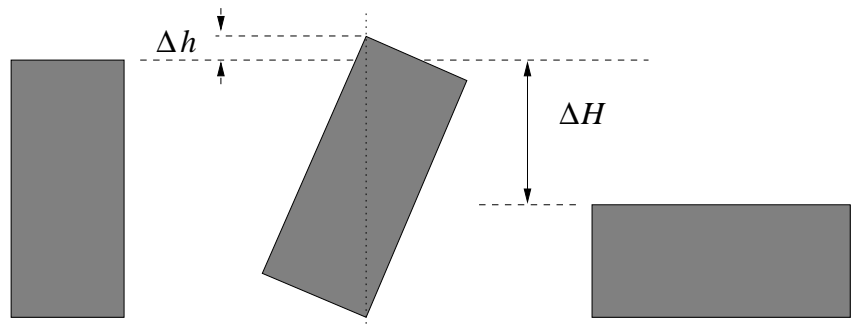

Fig. 1 - A vertical grain needs to be tilted through the height $\Delta h$ to reach the unstable equilibrium position and flop to the horizontal, while a horizontal grain needs to be tilted through an additional height $\Delta H$ to reach the vertical.

- If lattice sites $(i+1, j-1),(i+1, j)$, or $(i+1, j+1)$ are empty, grain $(i, j)$ moves there with a probability $\exp (-1 / \Gamma)$, in units such that the acceleration due to gravity, the mass of a grain, and the height of a lattice cell all equal unity.

- If the lattice site $(i-1, j)$ below the grain is empty, it will fall down.

- If lattice sites $(i-1, j \pm 1)$ are empty, the grain at height $h_{i j}$ will fall to either lower neighbour, provided the height difference $h_{i j}-h_{i-1, j \pm 1} \geq 2$.

- The grain flips from horizontal to vertical with probability $\exp \left(-m_{i j}(\Delta H+\Delta h) / \Gamma\right)$, where $m_{i j}$ is the mass of the pile (consisting of grains of unit mass) above grain $(i, j)$. For a rectangular grain, $\Delta H=1-a$ is the height difference between the initial horizontal and the final vertical state of the grain. Similarly, the activation energy for a flip reads $\Delta h=b-1$, where $b=\sqrt{1+a^{2}}$ is the diagonal length of a grain.

- The grain flips from vertical to horizontal with probability $\exp \left(-m_{i j} \Delta h / \Gamma\right)$.

Hereafter we consider $\Delta H$ and $\Delta h$ as phenomenological parameters, which will be seen to be related to the two main characteristic lengths in the system.

In line with recent investigations of compaction [1, 6, 10, 11], we examine the behaviour of the packing fraction of our model, as a function of the vibration intensity $\Gamma$. Let $N^{-}$and $N^{+}$ be the numbers of vertical and horizontal grains in the box. The packing fraction $\phi$ is:

$$
\phi=\frac{N^{+}-a N^{-}}{N^{+}+a N^{-}},
$$

which we use as an order parameter reflective of the behaviour of the compactivity 11. The vertical orientation of a grain thus wastes space proportional to $1-a$, relative to the horizontal one.

We examine the response of the packing fraction for $\Delta H=0.3, \Delta h=0.05$ to shaking at varying intensities in Fig. 2. Since the 'equilibrium' packing fraction $\phi_{\infty}$ (which we determined in separate runs) decreases with increasing intensity [6], we plotted the difference $\phi-\phi_{\infty}$ as a (logarithmic) function of time $T$ in the figure, starting with the same initial packing fraction in each case.

The dynamical response of the shaken sand-box includes three distinct regions, each illustrated by representative curves in the figure:

- a fluidised region (for $\Gamma \gg 1$ ), where we observe an initial increase (caused by a nonequilibrium and transient 'ordering' of grains in the boundary layer) of the packing 


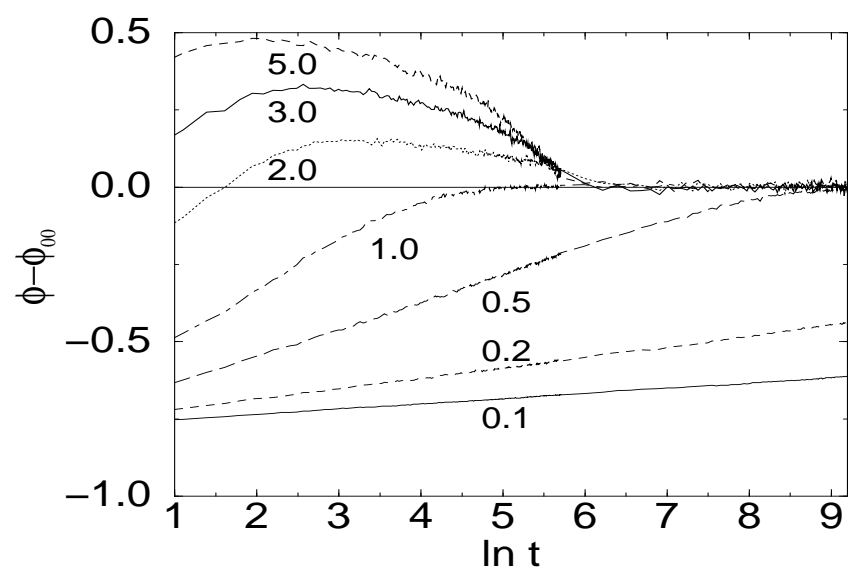

Fig. 2 - Plot of $\phi-\phi_{\infty}$ versus $\ln t$, for different values of $\Gamma$, indicated on the curves. Note that $\phi_{\infty}$ decreases with increasing $\Gamma$, and is thus distinct for each curve.

fraction that quickly relaxes to the equilibrium values $\phi_{\infty}$ in each case. This overshooting effect in Fig. 2 increases with $\Gamma$, since grains ever deeper in the sand-box can now overcome their activation energy to relax to the horizontal. This inhomogeneous relaxation has been seen in earlier, off-lattice simulations of compaction [12]. Analogous effects have also been observed in Ref. [13].

- an intermediate region (for $\Gamma \approx 1$ ), where the packing fraction remains approximately constant in the bulk, while the surface equilibrates via the fast dynamics of single-particle relaxation. The specific $\phi_{\infty}$ at which this occurs (0.917 here), is the single-particle relaxation threshold density observed in Ref. 13]; non-equilibrium, non-ergodic, fast dynamics allows single particles locally to find their equilibrium configurations at this density. Analogous effects have been observed in recent experiments on colloids [14], where the correlated dynamics of fast particles was seen to be responsible for most relaxational behaviour before the onset of the glass transition.

- a frozen region (for $\Gamma \ll 1$ ), where the slow dynamics of the system results in a logarithmic growth of packing fraction with time:

$$
\phi-\phi_{\infty}=b(\Gamma) \ln t+a,
$$

where $b(\Gamma)$ increases with $\Gamma$, in good agreement with experiment [1]. The slow dynamics has been identified [13] with a cascade process, where the free volume released by the relaxation of one or more grains allows for the ongoing relaxation of other grains in an extended neighbourhood. It includes the phenomenon of bridge collapse, which, for low vibration intensities, has been seen to be a major mechanism of compaction [6]. As $\Gamma$ decreases, the corresponding $\phi_{\infty}$ increases asymptotically towards the jamming limit $\phi_{\mathrm{jam}}$, identified with a dynamical phase transition in related work [13].

We next investigate the analogue of 'annealed cooling', where $\Gamma$ is increased and decreased cyclically, and the response of the packing fraction observed [1. The results obtained here are similar to those [12] seen using more realistic models of shaken spheres, but the simplicity of the present model allows for greater transparency. 


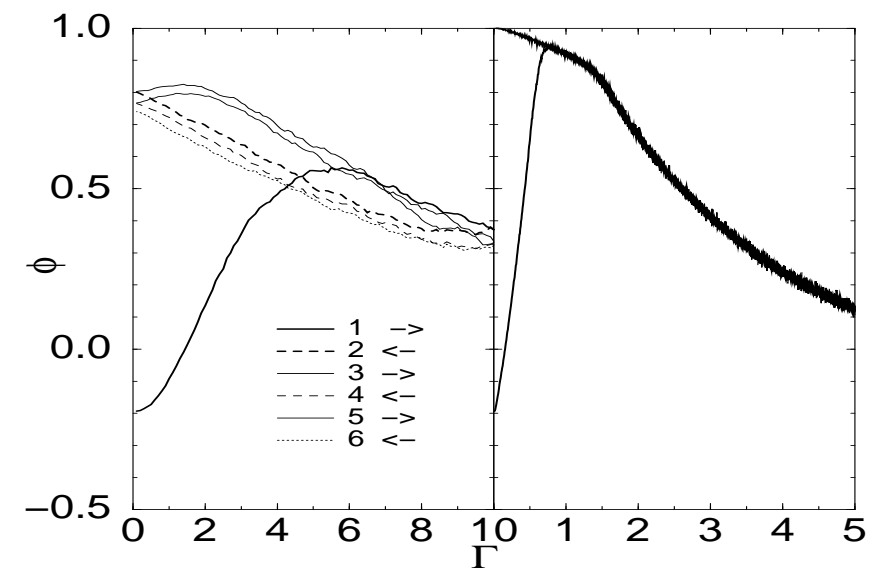

Fig. 3 - Hysteresis curves. Left: $\Delta \Gamma=0.1, t_{\text {tap }}=2000$ time units. Right: $\Delta \Gamma=0.001, t_{\text {tap }}=10^{5}$ time units. Note the approach of the irreversibility point $\Gamma^{*}$ to the 'shoulder' $\Gamma_{\mathrm{jam}}$, as the ramp rate $\delta \Gamma / t_{\text {tap }}$ is lowered.

Starting with the sand in a fluidised state, as in the experiment [1], we submit the sand-box to taps at a given intensity $\Gamma$ for a time $t_{\text {tap }}$ and increase the intensity in steps of $\delta \Gamma$; at a certain point, the cycle is reversed, to go from higher to lower intensities. The entire process is then iterated twice. Figure 3 shows the resulting behaviour of the volume fraction $\phi$ as a function of $\Gamma$, where an 'irreversible' branch and a 'reversible' branch of the compaction curve are seen, which meet at the 'irreversibility point' $\Gamma^{*}[1]$. The left- and right-hand side of Figure 3 correspond respectively to high and low values of the 'ramp rate' $\delta \Gamma / t_{\text {tap }}$ [1]. As the ramp rate is lowered, we note that:

- the width of the hysteresis loop in the so-called reversible branch decreases. The 'reversible' branch is thus not reversible at all; more realistic simulations of shaken spheres [6] confirm the first-order, irreversible nature of the transition, which allows the density to attain values that are substantially higher than random close packing, and quite close to the crystalline limit [2]. Precisely such a transition has also recently been observed experimentally in the compaction of rods [15].

- the 'irreversibility point' $\Gamma^{*}$ approaches $\Gamma_{\text {jam }}$ (the shaking intensity at which the jamming limit $\phi_{\mathrm{jam}}$ is approached), in agreement with results on other discrete models [16].

The simplicity of our model also permits us to explore the onset of 'glassy' behaviour between the regimes where fast and slow dynamics respectively predominate. We explore this via a configurational overlap function

$$
\chi\left(t_{\mathrm{ref}}, \Delta t\right)=\frac{1}{N} \sum_{i, j} \Theta\left[B_{i, j}\left(t_{\mathrm{ref}}\right), B_{i, j}\left(t_{\mathrm{ref}}+\Delta t\right)\right] .
$$

Here $B_{i, j}(t)$ can take three distinct values depending on whether the lattice site $(i, j)$ at time $t$ is (a) empty, (b) occupied by a + grain, (c) occupied by a - grain. We write $\Theta[X, Y]=$ $1-\delta_{X, Y}$; i.e., $\Theta[X, Y]=0$ if $X=Y . \Delta t$ is the time lag. Figure $\Theta$ shows results for different values of $\Gamma$, for $\Gamma=0.1,0.7,5$.

The left-hand panel $(\Gamma=0.1)$ shows the logarithmic behaviour characteristic of ageing, while the right-hand panel $(\Gamma=5)$ shows the quick equilibration virtually independent of 


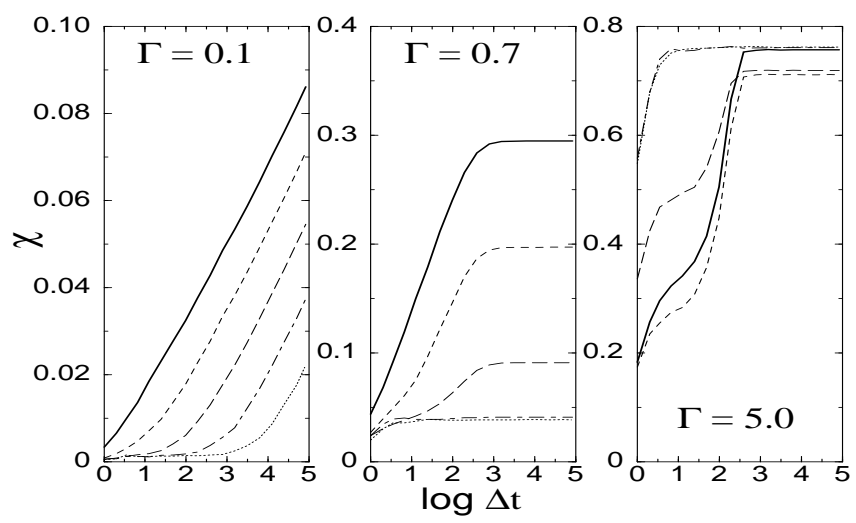

Fig. 4 - Overlap functions $\chi\left(t_{\mathrm{ref}}, \Delta t\right)$, equation (3), for $\Gamma=0.1,0.7,5$. Line styles distinguish five reference times from $t_{\text {ref }}=1$ (full line) to $t_{\text {ref }}=10^{4}$ (dotted line). The time unit is defined as $H W$ attempted Monte Carlo moves.

waiting times, which characterises the fluidised regime. The middle panel $(\Gamma=0.7)$ exemplifies the behaviour characteristic of the transition between the two regimes: there is an apparent 'equilibration' into different metastable states depending on the waiting time $t_{\mathrm{ref}}$.

The frozen regime is characterised by an absence of holes within the sand-box, and negligible surface roughness. Here, our model reduces to an exactly solvable model of $W$ independent columns of $H$ noninteracting 'grains' $\sigma_{n}(t)= \pm 1$, with $\sigma=+1$ denoting a horizontal grain, and $\sigma=-1$ denoting a vertical grain. The orientation of the grain at depth $n$, measured from the top of the system, evolves according to a Markov dynamics with depth-dependent rates

$$
\left\{\begin{array}{l}
w(-1 \rightarrow+1)=\exp (-n \Delta h / \Gamma) \\
w(+1 \rightarrow-1)=\exp (-n(\Delta H+\Delta h) / \Gamma)
\end{array}\right.
$$

as $m_{i j}=n=H+1-i$.

The order parameter describing the mean orientation, which we hereafter refer to as 'orientedness', $\bar{M}(t)=(1 / H) \sum_{n=1}^{H} M_{n}(t)$, with $M_{n}(t)=\left\langle\sigma_{n}(t)\right\rangle$, is related to the packing fraction of equation (1) as

$$
\bar{M}=\frac{(1+a) \phi-(1-a)}{1+a-(1-a) \phi} .
$$

At equilibrium, the orientedness profile is given by $M_{n, \text { eq }}=\tanh \left(n /\left(2 \xi_{\text {eq }}\right)\right)$, while the local equilibration time diverges exponentially with depth $n$, as $\tau_{n \text {,eq }} \approx \exp \left(n / \xi_{\text {dyn }}\right)$. These expressions involve two characteristic lengths of the model, the equilibrium length $\xi_{\text {eq }}$ and the dynamical length $\xi_{\text {dyn }}$, which read

$$
\xi_{\mathrm{eq}}=\frac{\Gamma}{\Delta H}, \quad \xi_{\mathrm{dyn}}=\frac{\Gamma}{\Delta h} .
$$

In the scaling regime where the height $H$ and both lengths $\xi_{\text {eq }}, \xi_{\text {dyn }}$ are large, the mean orientedness is $\bar{M}_{\text {eq }} \approx\left(2 \xi_{\text {eq }} / H\right) \ln \cosh \left(H /\left(2 \xi_{\text {eq }}\right)\right)$. For $H \ll \xi_{\text {eq }}, \bar{M}_{\text {eq }} \approx H /\left(4 \xi_{\text {eq }}\right) \ll 1$ : the system is very weakly ordered, even at equilibrium. For $H \gg \xi_{\text {eq }}, \bar{M}_{\text {eq }} \approx 1-(2 \ln 2) \xi_{\text {eq }} / H$ : the system is strongly ordered at equilibrium, except for its top skin layer, whose depth is of order $\xi_{\text {eq. }}$. 
TABLE I - Two different non-equilibrium regimes.

\begin{tabular}{lll}
\hline & Regime I & Regime II \\
\hline & $\xi_{\text {eq }} \ll \Lambda(t) \ll H$ & $\Lambda(t) \ll \xi_{\text {eq }}, H$ \\
& $(\omega \ln t \gg 1)$ & $(\omega \ln t \ll 1)$ \\
\hline $\bar{M}(t)$ & $\Lambda(t) / H$ & {$[\Lambda(2 t)]^{2} /\left(4 H \xi_{\text {eq }}\right)$} \\
$\bar{S}(t, s)$ & $1-[\Lambda(t)-\Lambda(s)] / H$ & $1-[\Lambda(2(t-s))] / H$ \\
$\bar{C}(t, s)$ & $1-[2 \Lambda(t)-\Lambda(t+s)] / H$ & $1-[\Lambda(2(t-s))] / H$ \\
\hline
\end{tabular}

As the equilibration time diverges exponentially with the depth, orientational order propagates down the system logarithmically slowly. More specifically, for a large but finite time $t$, only a top layer up to an 'ordering length' $\Lambda(t)$ has equilibrated, with

$$
\Lambda(t) \approx \xi_{\text {dyn }} \ln t .
$$

We have $M_{n}(t) \approx M_{n \text {,eq }}$ for $n \ll \Lambda(t)$, whereas $M_{n}(t) \approx 0$ for $n \gg \Lambda(t)$. The most ordered grains are situated at a depth comparable to $\Lambda(t)$; they have a maximum orientedness $M_{\max }(t) \approx \tanh ((\omega / 2) \ln t)$, where

$$
\omega=\frac{\xi_{\mathrm{dyn}}}{\xi_{\mathrm{eq}}}=\frac{\Delta H}{\Delta h}=\frac{1-a}{b-1}
$$

is the ratio of both characteristic lengths.

The length $\xi_{\text {eq }}$ is the length upto which disorder persists in the granular material when it has attained equilibrium. The length $\xi_{\text {dyn }}$ determines the length to which order has propagated in the granular material in the glassy regime. For grains where there are nearly equivalent orientations, even at equilibrium, there will be a large number of 'disordered' configurations in the top layer, since these will be almost equivalent to the strictly ordered one. (An extreme example would be $a \rightarrow 1$, which approximately corresponds to the packing of spheres, which are known to be disordered even in the nominally 'equilibrium' state of random close packing.) The length $\xi_{\mathrm{dyn}}$ controls the rate at which order propagates as a function of time in the glassy regime of a compacted powder. Both lengths, in experimental terms, thus have the interpretation of the depth of the boundary layer in a vibrated granular system; in the first case, this description applies when equilibrium has been reached, while in the second case, this applies to the nonequilibrium evolution of a vibrated granular bed.

In order for the model to exhibit interesting non-equilibrium or ageing effects, one must have $\Lambda(t) \ll H$. The two-time quantities we investigate to explore ageing are the full two-time correlation function, $S_{n}(t, s)=\left\langle\sigma_{n}(t) \sigma_{n}(s)\right\rangle$, and the connected one, $C_{n}(t, s)=S_{n}(t, s)-$ $M_{n}(t) M_{n}(s)$, with $0 \leq s$ (waiting time) $\leq t$ (observation time). In terms of the overlap function of equation (3), we have $\bar{S}\left(t_{\text {ref }}+\Delta t, t_{\text {ref }}\right)=1-2 \chi\left(t_{\text {ref }}, \Delta t\right)$. We are led to consider two different non-equilibrium regimes. In each case, the mean observables can be expressed, after some algebra, in terms of the ordering lengths only (see Table I). In Regime I, the maximal ordering is very close to perfect, as $1-M_{\max }(t) \sim t^{-\omega} \ll 1$. This is the conventional frozen regime (to which our data in Figure 1 correspond). The top layer of the system is strongly ordered, most of the grains are flat, and likely to stay that way: the ageing phenomenon corresponds to the slow ordering attempts of grains deeper in the bulk, quantified by the logarithmic growth of the ordering length $\Lambda(t)$. Table 1 shows that the mean orientedness is nothing but the fraction $\Lambda(t) / H$ of the system that has equilibrated. The two-time correlations are non-stationary, and they involve $\Lambda(s), \Lambda(t)$, and $\Lambda(t+s)$. 
In Regime II, the maximal ordering is very weak, as $M_{\max }(t) \approx(\omega / 2) \ln t \ll 1$. This regime exists only for $\omega \ll 1$, i.e., $a \rightarrow 1$ in the geometrical model. It corresponds to an even slower dynamics, since now any attempts at ordering are hindered additionally by a strong probability that a horizontal grain will flip to the vertical orientation. Table 1 shows that the mean orientedness involves the square of the ordering length, while the two-time correlations do not exhibit any non-stationary features characteristic of ageing, at least to leading order, in this scaling regime.

The physical difference between the two scenarios is comprehensible in terms of disorder in grain shapes. Where grains are very irregularly shaped (Regime I), the non-equilibrium regime will carry all the usual characteristics of ageing. Where, however, grains are regularly shaped (Regime II), the signatures of ageing will be hard to detect even in a highly non-equilibrium regime. It would be interesting to test this experimentally: would ageing experiments carried out separately on weakly vibrated rods (Regime I) or spheres (Regime II) have different results?

In conclusion, the simplicity of our model makes it a useful conceptual tool for probing the dynamical responses of vibrated sand, from the fluidised to the frozen regimes. In the latter case, our model is exactly solvable, which allows one to describe the by now well-established picture of logarithmic compaction, in terms of two characteristic lengths. The improvement of this necessarily qualitative picture of ageing by the addition of more realistic and complex interactions, while still retaining the overall conceptual simplicity of our model, constitutes the focus of current research.

\section{REFERENCES}

[1] E.R. Nowak, J.B. Knight, M. Povinelli, H.M. Jaeger, and S.R. Nagel, Powder Technology 94, 79 (1997); E.R. Nowak, J.B. Knight, E. Ben-Naim, H.M. Jaeger, and S.R. Nagel, Phys Rev. E 57, 1971 (1998).

[2] A. Mehta and G.C. Barker, J. Phys. Cond. Matt. 12, 6619 (2000).

[3] C. Campbell, Ann. Rev. Fluid Mech. 2, 57 (1994).

[4] S. McNamara and W.R. Young, Phys. Rev. E 50, R28 (1994); J.J. Brey, F. Moreno, and J.W. Dufty, Phys. Rev. E 54, 445 (1996); T.P.C. van Noije, M.H. Ernst, and R. Brito, Phys. Rev. E 57, R4891 (1998); M.L. Tan, I. Goldhirsch, Phys. Rev. Lett. 81, 3022 (1998); C. Bizon, M.D. Shattuck, J.B. Swift, and H.L. Swinney, Phys. Rev. E 60, 4340 (1999).

[5] M. Nicolas, P. Duru, and O. Pouliquen, Eur. Phys. J. E 3, 309 (2000).

[6] A. Mehta and G.C. Barker, Phys. Rev. Lett. 67, 394 (1991); Phys. Rev. A 45, 3435 (1992); Phys. Rev. E 47, 184 (1993).

[7] G.C. Barker and A. Mehta, Europhys. Lett. 27, 501, (1994); Phys. Rev. E 53, 5704 (1996); Physica A 283, 328 (2000); Phys. Rev. E 61, 6765 (2000).

[8] J. Kurchan, J. Phys. Cond. Matt. 12, 6611 (2000).

[9] L. Berthier, L.F. Cugliandolo, and J.L. Iguain, Phys. Rev. E 63, 051302 (2001).

[10] Th. Boutreux, E. Raphaël, and P.G. de Gennes, Phys. Rev. E 58, 4692 (1998); A. Aradian, E. Raphaël, and P.G. de Gennes, Phys. Rev. E 60, 2009 (1999); P.G. de Gennes, J. Coll. Int. Sci. 226, 1 (2000).

[11] S.F. Edwards, in Granular Matter: An Interdisciplinary Approach, ed. A. Mehta (Springer, New York, 1994); S.F. Edwards and D.V. Grinev, Phys. Rev. E 58, 4758 (1999).

[12] G.C. Barker and A. Mehta, preprint cond-mat/0010268 (2000).

[13] J. Berg and A. Mehta, preprint cond-mat/0012416 (2000).

[14] E. Weeks, J.C. Crocker, A.C. Levitt, A. Schofield, and D.A. Weitz, Science 287, 627 (2000).

[15] F. Villarruel, B.E. Lauderdale, D.M. Mueth, and H.E. Jaeger, Phys. Rev. E 61, 6914 (2000).

[16] A. Coniglio and M. Nicodemi, J. Phys. Cond. Matt. 12, 6601 (2000). 